\title{
Impact of vitamin D supplementation on health-care use in a 25-hydroxyvitamin D-tested population in France: a population-based descriptive cohort study
}

\author{
Pascal Caillet ${ }^{1,2,3, *}$, Susan B Jaglal ${ }^{4}$, Laurent Laforest ${ }^{2,5,6}$, Roland Chapurlat $^{2,3,7}$, \\ Muriel Rabilloud ${ }^{2,8,9}$, Michel Ducher ${ }^{10}$ and Anne-Marie Schott ${ }^{1,2,3}$ \\ 'Hospices Civils de Lyon, Pôle Information Médicale Evaluation, Recherche, Clinical Epidemiology Group, Lyon, \\ F-69003 France: ${ }^{2}$ Lyon 1 University, Faculty of Medicine, Lyon, France: ${ }^{3}$ INSERM, U1033, Physiopathology, \\ Diagnosis and treatment of Bone Diseases, Epidemiological and Clinical approaches of Bone Diseases' Group, Lyon, \\ France: ${ }^{4}$ University of Toronto, Department of Physical Therapy, Toronto, Ontario, Canada: ${ }^{5}$ Hospices Civils de Lyon, \\ Pharmacoepidemiology Unit, Lyon, France: ${ }^{6} \mathrm{CNRS}$, UMR 5558, LBBE, Evaluation and Modeling with the \\ Therapeutic Effects Group, Villeurbanne, France: ${ }^{7}$ Hospices Civils de Lyon, Rheumatology, Lyon, France: ${ }^{8}$ Hospices \\ Civils de Lyon, Biostatistics Service, Lyon, France: ${ }^{9}$ CNRS, UMR 5558, LBBE, Biostatistics - Health Group, \\ Villeurbanne, France: ${ }^{10}$ Hospices Civils de Lyon, Geriatric Hospital Group, Francheville, France
}

Submitted 16 July 2013: Final revision received 13 February 2014: Accepted 27 February 2014: First published online 31 March 2014

\begin{abstract}
Objective: Chronic vitamin D deficiency has been associated in some patients with diffuse musculoskeletal pain. These unspecific symptoms may partly explain why vitamin D deficiency is often diagnosed late. Our aim was to analyse health-care claims after vitamin D supplementation in patients likely to have vitamin D deficiency.

Design: Ambulatory health-care claims were compared before and after a vitamin D supplementation prescribed following a 25-hydroxyvitamin D assay.

Setting: Health Insurance Fund (FHIF) database of the Rhône-Alpes area, France. Subjects: Among patients reimbursed for a 25-hydroxyvitamin D assay between 1 December 2008 and 31 January 2009, those supplemented with vitamin D after the assay were matched on the date of assay to patients who did not receive vitamin D.

Results: Among the 3023 patients who had a 25-hydroxyvitamin D assay, 935 were consequently supplemented and matched to 935 patients not supplemented. Their median age was $50 \cdot 0$ and $49 \cdot 5$ years, respectively. Patients supplemented decreased their muscle relaxant consumption whereas no change was observed in the reference group, the difference between the two groups was significant $(P=0.03)$. Second and third Pain Relief Ladder prescriptions decreased in both groups but not significantly differently between groups $(P=0 \cdot 58)$. There was a decrease in prescriptions of biological examination in both groups with no significant difference.

Conclusions: Besides a decrease in muscle relaxant prescriptions in the supplemented group, it was difficult to assess the impact of vitamin D supplementation in patients likely to have vitamin D deficiency. Prospective cohort studies and randomized trials are needed to assess the efficiency of screening and supplementing vitamin D deficiency.
\end{abstract}

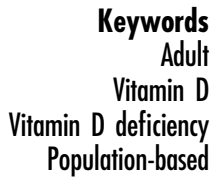

It is well known from clinical studies that severe chronic vitamin D deficiency in adults causes osteomalacia, a bone condition characterized by bone pain, muscle weakness and fracture (either microfractures or transcortical fractures $)^{(1)}$. A number of articles have occasionally reported limited series of undiagnosed cases of osteomalacia in individuals with vitamin D deficiency due to either nutritional reasons (e.g. gastrointestinal disorder and/or bariatric surgery) or a total absence of sun exposure (e.g. veiled women $)^{(2,3)}$. When patients present with symptoms such as chronic bone pain, muscle weakness or fracture, osteomalacia is rarely suspected by the referring physician, and the lag between the development of symptoms and the vitamin D deficiency diagnosis may be long ${ }^{(4)}$. It is possible that less severe but chronic vitamin D deficiency may cause comparable symptoms, although the 
25-hydroxyvitamin D (25(OH)D) level at which symptoms may arise is not precisely known and probably highly variable across individuals ${ }^{(1)}$. Additionally, vitamin $\mathrm{D}$ deficiency has been found to be associated with increased incidence of fractures, falls, neuromuscular and endocrinal impairments, and chronic pain in elderly populations ${ }^{(5-7)}$. However, very few studies have been conducted in healthy younger adults regarding the possible association between $25(\mathrm{OH}) \mathrm{D}$ levels and negative health outcomes although a significant proportion have vitamin D deficiency ${ }^{(5,8-11)}$. These studies also found that the most deficient individuals had not been previously diagnosed. Other studies suggested that the delay in vitamin D deficiency diagnosis generates over-use of inappropriate health-care resources prior to the deficiency being diagnosed and treated ${ }^{(4,12-15)}$. Based on these hypotheses, we could expect that in patients with no severe co-morbidities in a primary care setting, when vitamin D deficiency is diagnosed and treated by vitamin D supplementation, it is associated with a reduction in health-care use thereafter. The purpose of the present study was to analyse health-care use before and after a prescription of vitamin D supplementation following a 25(OH)D assay in a young to middle-aged population and to compare it with that in a non-supplemented group regarded as a reference group.

\section{Experimental method}

\section{The French Health Insurance Funds}

France has a publicly funded health system that systematically covers the population ${ }^{(16)}$. Briefly, maternity/ sickness and paternity insurance benefits are provided by the local Health Insurance Funds (Caisses Primaires d'Assurance Maladie) and $99.9 \%$ of the French population is covered with a public insurance (i.e. 63 million individuals). Reimbursements relate to all medical procedures given to the patients: visits to health-care professionals, drugs, biological and medical procedures.

\section{Drug reimbursement in the French Health Insurance Funds}

France has still a pharmaceutical monopoly; most drugs are only available in pharmacies (community or hospital), with a mandatory medical prescription. Drugs containing high doses of vitamin D (e.g. $2500 \mu \mathrm{g}$ (100000 IU) ampoules) must be prescribed before dispensation and are reimbursed on a $65 \%$ regular basis.

\section{Study design and study population}

We conducted a before-after analysis of health-care use in a cohort of individuals who had a $25(\mathrm{OH}) \mathrm{D}$ assay and a vitamin $\mathrm{D}$ prescription after the assay and compared it with that in the reference group of individuals who did not have a vitamin D prescription after the assay. We used data from the Rhône-Alpes area (6 million inhabitants in 2009) provided by the ERASME database (Extraction, Recherches, Analyses pour un Suivi Médico-Economique) ${ }^{(17)}$, a regional component of the French Health Insurance Funds (FHIF).

Patients aged 13-60 years who had a 25(OH)D assay between 1 December 2008 and 31 January 2009 were selected. Patients presenting with one of the thirty severe chronic diseases classified as 'affection de longue durée' $(A L D)^{(18)}$ were excluded, because people suffering severe chronic diseases are different from the general population regarding their health-care use. The list of diseases giving access to the ALD programme is currently defined. These chronic diseases include: stroke, bone marrow failure and other chronic cytopenia, chronic arteriopathy with ischaemic manifestations, complicated bilharziasis, severe heart diseases, chronic active liver disease and cirrhosis, primary or HIV acquired immunodeficiency, type I or II diabetes, severe neuromuscular disease (including myopathy and severe epilepsy), severe and chronic haemoglobinopathy, haemophilia, severe arterial hypertension, CHD, chronic respiratory failure, Alzheimer's disease, Parkinson's disease, metabolic inherited disease, cystic fibrosis, severe and chronic renal disease, paraplegia, vasculitides, systemic lupus erythematosus, scleroderma, rheumatoid arthritis, long-term psychiatric disease, chronic ulcerative colitis and Crohn's disease, multiple sclerosis, evolutive scoliosis, severe spondylarthritis, organ transplant consequences, tuberculosis, leprosy and cancer diseases. This programme involves about $16 \%$ of the patients insured by the FHIF. Patients with a vitamin D dispensation during the 5-month period preceding the assay, who died, or who were assigned to another healthcare insurance system during the follow-up period were also excluded.

\section{Outcome and factors associated with vitamin $D$ deficiency}

Individuals were considered as vitamin $\mathrm{D}$ deficient if they had a record of $25(\mathrm{OH}) \mathrm{D}$ assay followed by vitamin $\mathrm{D}$ supplementation (i.e. at least one occurrence of ergocalciferol (vitamin $\mathrm{D}_{2}$ ) or cholecalciferol (vitamin $\mathrm{D}_{3}$ ) reimbursement over the 3 months after the assay). They are referred to as the supplemented group. Patients who were not prescribed vitamin $\mathrm{D}$ after their assay were considered as not $25(\mathrm{OH}) \mathrm{D}$ deficient, and were regarded as the reference group. Each patient of the reference group was matched with a patient of the supplemented group on $25(\mathrm{OH}) \mathrm{D}$ assay date, within a range of $5 \mathrm{~d}$. The index date for each pair was the date of supplementation for the supplemented patient. Health-care use for every patient was recorded over two 5-month periods. Five months was chosen for the following reasons. First, the French Insurance Healthcare database is subject to regular turnover; only 24 months' data are stored, and each month, when the data of the newest month are entered in the system, those of the oldest month disappear. Second, we did not include in the analysis the period between the 
assay and the first prescription of vitamin $D$ because no change in health-care use was expected during this period. Third, the last three months of the 24-month database is not as reliable as the preceding months because the data regarding prescriptions are entered in the system only at the time of the reimbursement. Although for most patients the reimbursement is done automatically when purchasing drugs with their health insurance card (carte 'VITAL') from the pharmacy, a minority (those who do not have their card when purchasing the drugs) do not appear in the database until they send their claims by mail. For comparing health-care use between pre- and post-supplementation periods, we extracted the following information from the database: age, sex, number of physician visits and recorded medical interventions (according to the French classification of medical procedures), different drug prescriptions, drug classes (defined by Anatomical Therapeutical Chemical (ATC) codes $^{(19)}$ ) per distinct dates of prescription, medical imaging examinations, incident sick leaves and hospitalizations. The medical procedures were also classified as therapeutic or diagnostic. We identified and extracted reimbursements for specific drugs or biological examinations that were expected to be prescribed in patients presenting with vitamin D deficiency symptoms, such as diffuse musculoskeletal pain, asthenia or apparent depression. Regarding drugs, we considered analgesics of the second and third rung of the WHO's Pain Relief Ladder (PRL $)^{(20,21)}$, muscle relaxants, corticosteroids, thyroid hormones, iron-based preparations and antidepressants. Analgesics corresponding to the first rung of the PRL were not considered as they are too highly prone to over-the-counter use, and consequently estimation of their use would be strongly biased. Antiepileptic drugs were abstracted as they may be responsible for a vitamin $\mathrm{D}$ deficiency. We analysed claims for the following biological examinations: cell blood counts, transaminase level, serum creatinine and thyroid-stimulating hormone level, since they are expected to be prescribed in the case of vitamin D deficiency symptoms such as unspecific musculoskeletal pain.

\section{Statistical analysis}

The study population was described using means and standard deviations for continuous variables and frequencies and proportions for discrete variables. The supplemented and the reference groups were compared using the $t$ test when variables were continuous and the $\chi^{2}$ test when variables were discrete. Health-care use within each group was compared before and after the index date using a paired $t$ test on the same sample for continuous variables, McNemar's $\chi^{2}$ test for binomial discrete variables and Bowker's test for discrete variables with more than two categories. A $P$ value of 0.05 or less was considered statistically significant. Analyses were performed using the SAS Enterprise Guide version $4 \cdot 3$.

\section{Results}

\section{Characteristics of the supplemented group}

Over the two-month inclusion period (1 December 2008-31 January 2009), 3023 patients aged 13-60 years were identified (Fig. 1). Their mean age was 47.5 (SD 11.0) years, their median age was $49 \cdot 8$ years and $84.1 \%$ were women. The first assay recorded was a $25(\mathrm{OH}) \mathrm{D}_{3}$ assay in almost all cases (98.5\%). Among these patients, $45.2 \%$ (n 1367) received vitamin $\mathrm{D}$ supplementation following the assay and 935 of them were matched to the 935 controls who did not receive any $25(\mathrm{OH}) \mathrm{D}$ during the whole follow-up period. The mean age of the supplemented population was 46.9 (sD 10.8) years, their median age was 50.0 years and $85.1 \%$ were women. Baseline characteristics are shown in Table 1 . Half of the patients were 50-60 years old, $26 \cdot 1 \%$ were $40-50$ years old and $23.9 \%$ were 13-40 years old. Most patients (66.4\%) had up to two different classes of drugs prescribed over the 5-month baseline period before the 25(OH)D assay and 9.5\% had at least one sick leave. Thirteen per cent of patients were prescribed muscle relaxants, $15.7 \%$ corticosteroids, and $23.2 \%$ a second or third PRL analgesic.

\section{Characteristics of the reference group}

Baseline characteristics of the reference group are shown Table 1. The supplemented group had higher baseline health-care use compared with the reference group as seen by the number of distinct pharmacy prescriptions (15.9\% with six or more prescriptions $v .9 .7 \%, P<0.001$ ), different ATC drug classes reimbursed (33.6\% with more than two classes per prescription $v .27 \cdot 7 \%, P=0 \cdot 01)$ and most of the study drugs $(P<0.05$ for antidepressants, corticosteroids, muscle relaxants and analgesics).

\section{Before and after comparisons}

In the supplemented group, $24 \%$ of the patients received less than $5000 \mu \mathrm{g}$ (200000 IU) over the 5-month follow-up, approximately $40 \%$ of the patients received between 5000 and $10000 \mu \mathrm{g}$ (200000 and $400000 \mathrm{IU})$ and 36\% received more than $10000 \mu \mathrm{g}$ (400000 IU). Prescriptions of drugs expected to be associated with vitamin D deficiency symptoms are shown in Table 2. In the supplemented group, fewer patients were treated by muscle relaxants after supplementation than before $(13.1 \% v .8 .6 \%, P<0.001)$. Such a pattern was not observed in the reference group, where there was no change in muscle relaxant prescription frequency $(10 \cdot 1 \% v \cdot 10 \cdot 7 \%, P=0 \cdot 68)$. Regarding second and third PRL analgesics, prescriptions tended to decrease in both groups although this did not reach statistical significance in the supplemented group $(23 \cdot 2 \% v .21 \cdot 2 \%, P=0 \cdot 19$ in supplemented group; $19.2 \% v \cdot 16.0 \%, P=0.04$ in reference group). There was no change in the number of specialists visited during the post-supplementation period compared with the pre-supplementation period in both groups. The overall number of pharmacy prescriptions per 


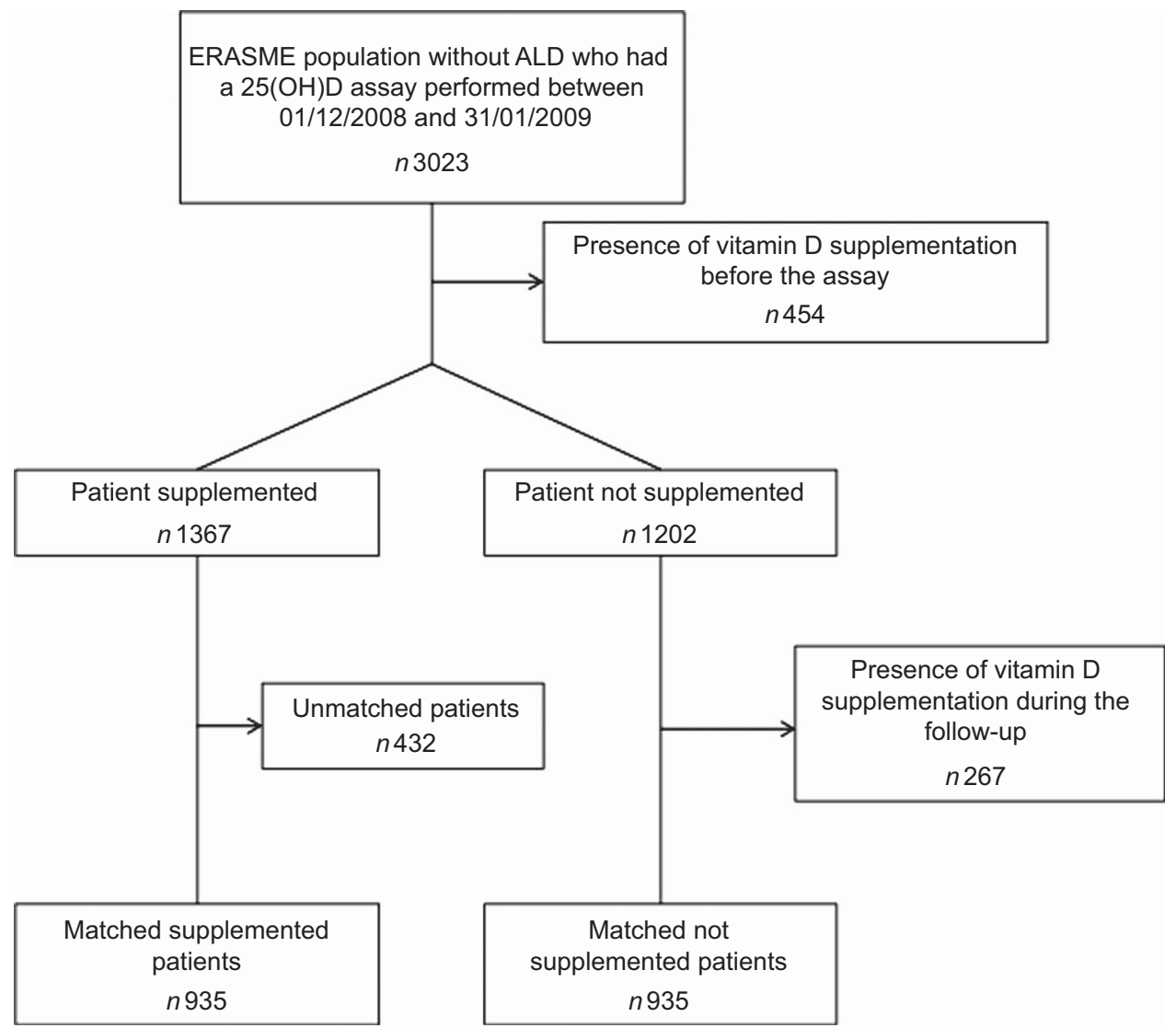

Fig. 1 Flowchart of the study (ERASME, Extraction, Recherches, Analyses pour un Suivi Médico-Economique (database); ALD, 'affection de longue durée'; 25(OH)D, 25-hydroxyvitamin D)

patient did not change significantly; neither did the number of sick leaves or medical imaging examinations per patient. The mean number of diagnosis-related procedures per patient did not change significantly. Between-group comparisons are displayed in Table 3. In the supplemented group, a higher proportion of patients decreased their use of muscle relaxants than in the reference group (10.2\% $v .7 .3 \%, P=0.03)$ and a lower proportion of patients decreased their use of thyroid hormones $(0.5 \% v .1 .6 \%$, $P=0.03)$. Regarding the supplemented group, we searched for whether there was a switch from muscle relaxants towards antidepressants, but we did not observe any statistically significant relationship between decrease in use of muscle relaxants and increase in use of antidepressants. For biological tests expected to be prescribed to patients with vitamin D deficiency symptoms, the supplemented group and reference group had similar decreasing patterns.

\section{Discussion}

In this population-based cohort of patients who had a 25$(\mathrm{OH}) \mathrm{D}$ assay during winter, we targeted a young to middleaged population aged 13-60 years who was free of heavy chronic disease and assessed global health-care use as well as specific prescriptions expected to be associated with vitamin D deficiency symptoms. We observed a moderate but statistically significant decrease in some prescriptions potentially associated with vitamin D deficiency symptoms i.e. pain and asthenia, particularly in muscle relaxant prescriptions. We also observed a decrease in prescriptions of second and third PRL analgesics but this was similar to the reference group. Besides, prescription of vitamin D supplementation in patients likely to have a vitamin D deficiency was not associated with a global decrease in the other health-care consumptions as measured by the number of physician visits or the number of diagnostic or therapeutic procedures performed.

It has been described in the literature that many patients with vitamin $\mathrm{D}$ deficiency may suffer from unspecific chronic fatigue or musculoskeletal pain and that the mean delay from symptoms to diagnosis of vitamin D deficiency is long ${ }^{(4,12-15)}$. In a before-after study conducted in Switzerland in 2005 among female asylum seekers with chronic complaints for bone pain, proximal muscular weakness, a change in gait and/or fatigue, the authors found a mean duration of symptoms of 2.5 years before a diagnosis of vitamin $\mathrm{D}$ deficiency was established. The mean number of emergency medical visits decreased by $44 \%$ in the sample after the diagnosis of vitamin D deficiency and vitamin D 
Table 1 Baseline characteristics of the study population and health-care use during the 5-month period preceding the $25(\mathrm{OH}) \mathrm{D}$ assay, overall and by further vitamin $\mathrm{D}$ supplementation status; data on young to middle-aged patients who had a $25(\mathrm{OH}) \mathrm{D}$ assay between 1 December 2008 and 31 January 2009, ERASME database (regional component of the FHIF), Rhône-Alpes area, France

\begin{tabular}{|c|c|c|c|c|c|c|c|}
\hline \multirow[b]{2}{*}{ Baseline characteristics/Health-care use } & \multicolumn{2}{|c|}{ Overall $(n$ 1870) } & \multicolumn{2}{|c|}{ Supplemented ( $n$ 935) } & \multicolumn{2}{|c|}{ Reference ( $n$ 935) } & \multirow[b]{2}{*}{$P$ value } \\
\hline & $n$ & $\%$ & $n$ & $\%$ & $n$ & $\%$ & \\
\hline Age (years) & & & & & & & 0.04 \\
\hline $13-<20$ & 64 & 3.4 & 32 & 3.4 & 32 & 3.4 & \\
\hline $20-<30$ & 126 & $6 \cdot 7$ & 46 & 4.9 & 80 & 8.6 & \\
\hline $30-<40$ & 283 & $15 \cdot 1$ & 146 & $15 \cdot 6$ & 137 & 14.7 & \\
\hline $40-<50$ & 481 & $25 \cdot 7$ & 244 & $26 \cdot 1$ & 237 & $25 \cdot 3$ & \\
\hline $50-60$ & 916 & $49 \cdot 0$ & 467 & $50 \cdot 0$ & 449 & $48 \cdot 0$ & \\
\hline Female gender & 1572 & $84 \cdot 1$ & 139 & 14.9 & 159 & $17 \cdot 0$ & 0.20 \\
\hline Number of pharmacy prescriptions & & & & & & & 0.001 \\
\hline$\leq 5$ & 1630 & $87 \cdot 2$ & 786 & $84 \cdot 1$ & 844 & $90 \cdot 3$ & \\
\hline $6-10$ & 221 & 11.8 & 140 & $15 \cdot 0$ & 81 & 8.7 & \\
\hline$>10$ & 19 & 0.1 & 9 & 0.9 & 10 & 1.0 & \\
\hline Number of different ATC $^{*}$ drug classes reimbursed per prescription & & & & & & & 0.01 \\
\hline$\leq 2$ & 1297 & 0.69 & 621 & 66.4 & 676 & $72 \cdot 3$ & \\
\hline $3-4$ & 491 & $26 \cdot 2$ & 273 & $29 \cdot 2$ & 218 & 23.3 & \\
\hline$>4$ & 82 & 4.4 & 41 & 4.4 & 41 & 4.4 & \\
\hline Number of different medical specialties visited & & & & & & & 0.21 \\
\hline$\leq 2$ & 1508 & 80.1 & 739 & $79 \cdot 0$ & 769 & $82 \cdot 2$ & \\
\hline $3-4$ & 309 & $16 \cdot 5$ & 168 & $18 \cdot 0$ & 141 & $15 \cdot 1$ & \\
\hline$>4$ & 53 & $2 \cdot 8$ & 28 & 3.0 & 25 & $2 \cdot 7$ & \\
\hline Presence of incident sick leaves & 184 & $9 \cdot 8$ & 89 & 9.5 & 95 & $10 \cdot 2$ & 0.64 \\
\hline Presence of medical imaging & 422 & $22 \cdot 6$ & 220 & 23.5 & 202 & $21 \cdot 6$ & 0.32 \\
\hline \multicolumn{8}{|l|}{ Presence of specific treatment reimbursed } \\
\hline Muscle relaxant & 218 & 11.6 & 123 & $13 \cdot 2$ & 95 & $10 \cdot 2$ & 0.04 \\
\hline Second and third PRL analgesics & 397 & 21.2 & 217 & $23 \cdot \overline{2}$ & 180 & 19.2 & 0.04 \\
\hline Corticosteroids & 260 & 13.9 & 147 & $15 \cdot 7$ & 113 & $12 \cdot 1$ & 0.02 \\
\hline Antidepressant agent & 240 & $12 \cdot 8$ & 135 & 14.4 & 105 & $11 \cdot 2$ & 0.04 \\
\hline Thyroid hormones & 145 & $7 \cdot 7$ & 74 & 7.9 & 71 & $7 \cdot 6$ & 0.79 \\
\hline Iron-based preparation & 45 & 2.4 & 24 & $2 \cdot 6$ & 21 & $2 \cdot 2$ & 0.65 \\
\hline Antiepileptic agent & 75 & 4.0 & 42 & 4.5 & 33 & 3.5 & 0.29 \\
\hline \multicolumn{8}{|l|}{ Presence of biological examinations } \\
\hline Blood count & 564 & $30 \cdot 2$ & 301 & $32 \cdot 2$ & 263 & $28 \cdot 1$ & 0.05 \\
\hline Serum creatinine & 438 & 23.4 & 236 & $25 \cdot 2$ & 202 & 21.6 & 0.06 \\
\hline Transaminase level & 321 & $17 \cdot 2$ & 169 & $18 \cdot 1$ & 152 & $16 \cdot 3$ & 0.30 \\
\hline Thyroid-stimulating hormone level & 422 & $22 . \overline{6}$ & 223 & 23.8 & 199 & $21 \cdot 3$ & 0.18 \\
\hline
\end{tabular}

25(OH)D, 25-hydroxyvitamin D; ERASME, Extraction, Recherches, Analyses pour un Suivi Médico-Economique; FHIF, French Health Insurance Funds; PRL, WHO's Pain Relief Ladder.

*Anatomical Therapeutic Chemical classification of drugs.

supplementation was established. Similarly, the mean number of analgesic drugs prescribed decreased by $51 \%$ after deficiency was treated ${ }^{(4)}$. In a previous study conducted by our university department of general practice in the Rhône-Alpes area in a cohort of 196 women with no chronic disease aged from 19 to 49 years, who wore concealing clothing and who consulted their general practitioner from January through March 2008, it was found that $95.9 \%$ of women had a $25(\mathrm{OH}) \mathrm{D}$ level $<75 \mathrm{nmol} / \mathrm{l}$ and $53.6 \%$ a level $<30 \mathrm{nmol} / \mathrm{l}$. Of all women studied, $53 \%$ had asthenia and $45 \%$ had musculoskeletal pain ${ }^{(3)}$. That study was consistent with other European studies showing that all these patients with unspecific musculoskeletal pain had not been screened for $25(\mathrm{OH}) \mathrm{D}$, and that 30-59 months had elapsed before the deficiency was diagnosed ${ }^{(3,22)}$.

In the present study we observed a significant decrease in muscle relaxant prescriptions in patients who had a 25$(\mathrm{OH}) \mathrm{D}$ assay followed by vitamin $\mathrm{D}$ supplementation in the 3 months. This decrease was significant whereas no decrease was observed in the reference group. Unfortunately, we could not assess the consumption of frequently used painkillers, as most of them are delivered over the counter. That is why we had to consider only the second and third PRL analgesics. A decrease in second and third PRL analgesic prescriptions was observed in both groups and thus could not be associated with the correction of vitamin D deficiency. However, chronic pain associated with longterm vitamin D deficiency might not often require second and third PRL analgesic prescriptions but mostly first PRL analgesics.

We observed an increase in the use of thyroid hormones and antidepressants after vitamin D supplementation. This result could be explained by the fact that vitamin $D$ deficiency investigation could be triggered by unspecific clinical signs like asthenia, which could be also due to hypothyroidism and depression. Therefore, an increase in use of pharmacotherapies indicated in hypothyroidism or depression after a $25(\mathrm{OH}) \mathrm{D}$ supplementation could signal a concomitant diagnosis of these diseases, associated with or caused by a vitamin D deficiency ${ }^{(23,24)}$. 
Table 2 Frequency of prescriptions of drugs and biological examinations expected to be associated with 25(OH)D deficiency symptoms in the two groups before and after the index date; data on young to middle-aged patients who had a 25(OH)D assay between 1 December 2008 and 31 January 2009, ERASME database (regional component of the FHIF), Rhône-Alpes area, France

\begin{tabular}{|c|c|c|c|c|c|}
\hline \multirow[b]{3}{*}{ Health-care use } & \multicolumn{5}{|c|}{ Presence of reimbursement for a given patient } \\
\hline & \multicolumn{2}{|c|}{ Before supplementation } & \multicolumn{2}{|c|}{ After supplemententation } & \multirow[b]{2}{*}{$P$ value } \\
\hline & $n$ & $\%$ & $n$ & $\%$ & \\
\hline \multicolumn{6}{|l|}{ Supplemented group ( $n$ 935) } \\
\hline \multicolumn{6}{|l|}{ Treatments reimbursed } \\
\hline Muscle relaxant & 123 & $13 \cdot 1$ & 80 & $8 \cdot 6$ & $<0.001$ \\
\hline Second and third PRL analgesics & 217 & 23.2 & 198 & $21 \cdot 2$ & 0.19 \\
\hline Corticosteroids & 147 & $15 \cdot 7$ & 131 & $14 \cdot 0$ & 0.26 \\
\hline Antidepressant agent & 135 & 14.4 & 163 & $17 \cdot 4$ & 0.005 \\
\hline Thyroid hormones & 71 & 7.6 & 94 & $10 \cdot 0$ & $<0.001$ \\
\hline Iron-based preparation & 24 & $2 \cdot 6$ & 31 & $3 \cdot 3$ & 0.34 \\
\hline Antiepileptic agent & 42 & 4.5 & 52 & $5 \cdot 6$ & 0.51 \\
\hline \multicolumn{6}{|l|}{ Biological examinations } \\
\hline Blood count & 301 & $32 \cdot 2$ & 235 & $25 \cdot 1$ & $<0.001$ \\
\hline Serum creatinine & 236 & $25 \cdot 2$ & 152 & $16 \cdot 3$ & $<0.001$ \\
\hline Transaminase level & 16 & $18 \cdot 1$ & 113 & $12 \cdot 1$ & $<0.001$ \\
\hline Thyroid-stimulating hormone level & 223 & 23.8 & 160 & $17 \cdot 1$ & $<0.001$ \\
\hline \multicolumn{6}{|l|}{ Reference group ( $n$ 935) } \\
\hline \multicolumn{6}{|l|}{ Treatments reimbursed } \\
\hline Muscle relaxant & 95 & $10 \cdot 1$ & 100 & $10 \cdot 7$ & 0.68 \\
\hline Second and third PRL analgesics & 180 & $19 \cdot 2$ & 150 & $16 \cdot 0$ & 0.04 \\
\hline Corticosteroids & 113 & $12 \cdot 1$ & 107 & 11.4 & 0.65 \\
\hline Antidepressant agent & 105 & 11.2 & 100 & $10 \cdot 7$ & 0.55 \\
\hline Thyroid hormones & 74 & $7 . \overline{9}$ & 74 & 7.9 & 1.00 \\
\hline Iron-based preparation & 21 & $2 \cdot 2$ & 28 & 3.0 & 0.26 \\
\hline Antiepileptic agent & 33 & 3.5 & 34 & $3 \cdot 6$ & 0.87 \\
\hline \multicolumn{6}{|l|}{ Biological examinations } \\
\hline Blood count & 263 & $28 \cdot 1$ & 179 & $19 \cdot 1$ & $<0.001$ \\
\hline Serum creatinine & 202 & 21.6 & 131 & 14.0 & $<0.001$ \\
\hline Transaminase level & 152 & $16 \cdot 2$ & 104 & $11 \cdot 1$ & $<0.001$ \\
\hline Thyroid-stimulating hormone level & 199 & 21.3 & 108 & 11.5 & $<0.001$ \\
\hline
\end{tabular}

25(OH)D, 25-hydroxyvitamin D; ERASME, Extraction, Recherches, Analyses pour un Suivi Médico-Economique; FHIF, French Health Insurance Funds; PRL, WHO's Pain Relief Ladder.

We did not observe an overall decrease in health-care use after $25(\mathrm{OH}) \mathrm{D}$ supplementation in patients likely to have a vitamin D deficiency. The total number of physician visits and therapeutic and diagnostic-related procedures did not decrease. There are several possible explanations for these observations. The main limitation of our study is the lack of information regarding $25(\mathrm{OH}) \mathrm{D}$ results in our administrative database. We assumed that patients who received supplementation after a $25(\mathrm{OH}) \mathrm{D}$ assay were deficient and that those who did not were not. However, we have no information on the degree of vitamin D deficiency and it is possible that some physicians may have overprescribed vitamin D. Nevertheless, if vitamin $\mathrm{D}$ was prescribed in non-deficient individuals no beneficial effect on health-care use may be expected in those patients. We assumed that clinicians who prescribed $25(\mathrm{OH}) \mathrm{D}$ assays, prescribed consequently vitamin $\mathrm{D}$ in case of vitamin D deficiency. However, it is possible that some patients have not complied with these prescriptions. Additionally, we cannot assess the appropriateness of the dose of vitamin D prescribed and some patients may not have received sufficient doses. Another explanation for our results is the limited time frame of the study. It is possible that 5 months was not enough to observe a significant effect of the supplementation, especially if the dose was inadequate. A final explanation would be that vitamin $\mathrm{D}$ supplementation has a limited effect on healthcare use ${ }^{(25)}$. We observed a significant decrease in muscle relaxant use and non-significant decrease in analgesic use subsequent to vitamin D supplementation, which was not associated with an increase of antidepressant use indicated in some cases of persistent neuropathic pain. There is emerging evidence on the efficacy of vitamin D to improve asthenia and/or pain in $25(\mathrm{OH}) \mathrm{D}$-deficient patients ${ }^{(26)}$ but the efficacy of vitamin D in pain relief is still debated. A Cochrane review from 2010 stated that the existing randomized controlled trials were too small to reach meaningful conclusions regarding the hypothesis of vitamin D supplementation as a treatment for chronic pain ${ }^{(27)}$.

\section{Conclusion}

Our findings only partially support our initial hypothesis that vitamin D supplementation of individuals with vitamin $\mathrm{D}$ deficiency decreases health-care use. We observed a decrease in myorelaxing drug claims only in the supplemented group $v$. the reference group, with no significant 
Table 3 Individual evolution of prescription of drugs and biological examinations expected to be associated with 25(OH)D deficiency symptoms; data on young to middle-aged patients who had a 25(OH)D assay between 1 December 2008 and 31 January 2009 , ERASME database (regional component of the FHIF), Rhône-Alpes area, France

\begin{tabular}{|c|c|c|c|c|c|c|}
\hline \multirow[b]{3}{*}{ Health-care use } & \multirow[b]{3}{*}{ Group } & \multicolumn{5}{|c|}{ Before-after evolution } \\
\hline & & \multicolumn{2}{|c|}{ Decrease in use } & \multicolumn{2}{|c|}{ No decrease in use } & \multirow[b]{2}{*}{$\chi^{2}$ test $P$ value } \\
\hline & & $n$ & $\%$ & $n$ & $\%$ & \\
\hline \multicolumn{7}{|l|}{ Treatments reimbursed } \\
\hline \multirow{2}{*}{ Muscle relaxant } & Supplemented & 95 & $10 \cdot 2$ & 840 & $89 \cdot 8$ & 0.03 \\
\hline & $\begin{array}{l}\text { Reference } \\
\text { Supplemented }\end{array}$ & $\begin{array}{r}68 \\
115\end{array}$ & $\begin{array}{r}7 \cdot 3 \\
12 \cdot 3\end{array}$ & $\begin{array}{l}867 \\
820\end{array}$ & $\begin{array}{l}92 \cdot 7 \\
87.7\end{array}$ & \\
\hline Second and third PRL analgesics & Reference & 123 & $\begin{array}{l}12 \cdot 3 \\
13 \cdot 2\end{array}$ & $\begin{array}{l}820 \\
812\end{array}$ & $\begin{array}{l}87 \cdot 7 \\
86 \cdot 8\end{array}$ & 0.58 \\
\hline \multirow[t]{2}{*}{ Corticosteroids } & Supplemented & 109 & $11 \cdot 2$ & 826 & $88 \cdot 3$ & 0.23 \\
\hline & Reference & 93 & 9.9 & 842 & $90 \cdot 0$ & \\
\hline \multirow[t]{2}{*}{ Antidepressant agent } & Supplemented & 35 & $3 \cdot 7$ & 900 & $96 \cdot 3$ & 0.81 \\
\hline & Reference & 37 & 3.9 & 898 & 96.0 & \\
\hline \multirow[t]{2}{*}{ Thyroid hormones } & Supplemented & 5 & 0.5 & 930 & 99.5 & 0.03 \\
\hline & Reference & 15 & 1.6 & 920 & 98.4 & \\
\hline \multirow{2}{*}{ Iron-based preparation } & Supplemented & 23 & 2.5 & 912 & 97.5 & 0.26 \\
\hline & Reference & 16 & $1 \cdot 7$ & 919 & 98.3 & \\
\hline \multirow[t]{2}{*}{ Antiepileptic agent } & Supplemented & 17 & 1.8 & 918 & 98.2 & 0.86 \\
\hline \multirow{2}{*}{\multicolumn{7}{|c|}{ Biological examinations }} \\
\hline & & & & & & \\
\hline \multirow[t]{2}{*}{ Blood count } & Supplemented & 201 & 21.5 & 734 & 78.5 & 0.65 \\
\hline & Reference & 209 & $22 \cdot 3$ & 726 & 77.6 & \\
\hline \multirow[t]{2}{*}{ Serum creatinine } & Supplemented & 180 & $19 \cdot 2$ & 755 & $80 \cdot 8$ & 0.15 \\
\hline & Reference & 156 & $16 \cdot 7$ & 779 & 83.3 & \\
\hline \multirow{2}{*}{ Transaminase level } & Supplemented & 139 & 14.9 & 796 & 85.1 & 0.43 \\
\hline & Reference & 127 & 13.6 & 808 & $86 \cdot 4$ & \\
\hline \multirow[t]{2}{*}{ Thyroid-stimulating hormone level } & Supplemented & 151 & $16 \cdot 1$ & 784 & 83.8 & 0.80 \\
\hline & Reference & 155 & $16 \cdot 6$ & 780 & 83.4 & \\
\hline
\end{tabular}

25(OH)D, 25-hydroxyvitamin D; ERASME, Extraction, Recherches, Analyses pour un Suivi Médico-Economique; FHIF, French Health Insurance Funds; PRL, WHO's Pain Relief Ladder.

switch towards other musculoskeletal pain pharmacotherapies. No overall decrease in diagnostic or therapeutic medical procedures was observed. Our study highlights potential areas for future research, including the frequency of undiagnosed vitamin D deficiency associated with diffuse musculoskeletal pain in the general population and the investigation of the efficacy of vitamin D supplementation on these symptoms in randomized controlled clinical trials.

\section{Acknowledgements}

Acknowledgements: The authors wish to thank Mrs V. Ambrosi, Dr R. Nublat and Dr G. Weill (French National Healthcare System), Professor C. Colin (Department of Medical Information) and Dr M.F. Le Goaziou (Department of General Practice). Financial support: This work was funded by French public institutions, i.e. the Hospices Civils de Lyon (HCL) and the Institut National de la Santé et de la Recherche Médicale (INSERM), which had no role in the design, analysis or writing of this article. The authors had full intellectual independence regarding the funders. Conflict of interest: All authors have completed the Unified Competing Interest form at www.icmje.org/coi_disclosure.pdf (available on request from the corresponding author) and declare that: (i) all authors have no support from companies for the submitted work; (ii) only R.C. had relationships with companies that might have an interest in the submitted work in the previous 3 years; (iii) the authors' spouses, partners or children have no financial relationships that may be relevant to the submitted work; and (iv) all authors have no nonfinancial interests that may be relevant to the submitted work. Authorship: P.C. was responsible for the conception and design, analysis and interpretation of data, and drafting the paper. S.J. assisted with interpretation of data and drafting the paper and revising it critically for important intellectual content. L.L. assisted with interpretation of data and drafting the paper and revising it critically for important intellectual content. R.C. assisted with interpretation of data, logistic resources management, and drafting the paper and revising it critically for important intellectual content. M.R. assisted with analysis and interpretation of data, and drafting the paper and revising it critically for important intellectual content. M.D. assisted with interpretation of data and drafting the paper and revising it critically for important intellectual content. A.-M.S. was responsible for the conception and design, analysis and interpretation of data, and drafting the paper and revising it critically for important intellectual content. Patients' consent to publication: Consent was not obtained but the presented data are anonymised and risk of identification is low. These data were used with approval of the National Informatic and Liberty Commission (CNIL). Access to the data: All authors had unrestricted access to the data. ENCEPP registration number: ENCEPP/SDPP/3260. 


\section{References}

1. Cianferotti L \& Marcocci C (2012) Subclinical vitamin D deficiency. Best Pract Res Clin Endocrinol Metab 26, 523-537.

2. Holick MF, Binkley NC, Bischoff-Ferrari HA et al. (2012) Guidelines for preventing and treating vitamin D deficiency and insufficiency revisited. J Clin Endocrinol Metab 97, 1153-1158.

3. Le Goaziou MF, Contardo G, Dupraz C et al. (2011) Risk factors for vitamin D deficiency in women aged 20-50 years consulting in general practice: a cross-sectional study. Eur J Gen Pract 17, 146-152.

4. de Torrenté de la Jara G, Pécoud A \& Favrat B (2006) Female asylum seekers with musculoskeletal pain: the importance of diagnosis and treatment of hypovitaminosis D. BMC Fam Pract $\mathbf{2 3}, 4$.

5. Annweiler C, Allali G, Allain P et al. (2009) Vitamin D and cognitive performance in adults: a systematic review. Eur J Neurol 16, 1083-1089.

6. Annweiler C, Schott AM, Berrut G et al. (2009) Vitamin D-related changes in physical performance: a systematic review. J Nutr Health Aging 13, 893-898.

7. Christakos S \& Deluca HF (2011) Minireview: Vitamin D: is there a role in extraskeletal health? Endocrinology 152, 2930-2936.

8. Tangpricha V, Pearce EN, Chen TC et al. (2002) Vitamin D insufficiency among free-living healthy young adults. $\mathrm{Am} \mathrm{J}$ Med 112, 659-662.

9. Chapuy MC, Preziosi P, Maamer M et al. (1997) Prevalence of vitamin D insufficiency in an adult normal population. Osteoporos Int 7, 439-443.

10. Daly RM, Gagnon C, Lu ZX et al. (2012) Prevalence of vitamin D deficiency and its determinants in Australian adults aged 25 years and older: a national, populationbased study. Clin Endocrinol (Oxf) 77, 26-35.

11. Forrest KYZ \& Stuhldreher WL (2011) Prevalence and correlates of vitamin D deficiency in US adults. Nutr Res $\mathbf{3 1}$, 48-54.

12. Nellen JF, Smulders YM, Jos Frissen PH et al. (1996) Hypovitaminosis D in immigrant women: slow to be diagnosed. BMI 312, 570-572.

13. Al-Said Y, Al-Rached H, Al-Qahtani H et al. (2009) Severe proximal myopathy with remarkable recovery after vitamin D treatment. Can J Neurol Sci 36, 336-339.

14. Lyman D (2005) Undiagnosed vitamin D deficiency in the hospitalized patient. Am Fam Physician 71, 299-304.
15. Fabbriciani G, Pirro M, Leli C et al. (2010) Diffuse muscoskeletal pain and proximal myopathy: do not forget hypovitaminosis D. J Clin Rheumatol 16, 34-37.

16. Caisse Nationale d'Assurance Maladie des Travailleurs Salariés (2013) Getting informed about health insurance. http://www.ameli.fr/l-assurance-maladie/connaitre-l-assurancemaladie/getting-informed-about-health-insurance/ (accessed January 2014).

17. Fender P \& Weill A (2004) Epidemiology, public health and medical rates databases. Rev Epidemiol Sante Publique 52 , 113-117.

18. Nolte E, Knai C, McKee M, on behalf of World Health Organization (2008) Managing chronic conditions. Experience in eight countries. http://www.euro.who.int/_data/ assets/pdf_file/0008/98414/E92058.pdf (accessed January 2014).

19. World Health Organization (2013) Guidelines for ATC classification and DDD assignment. http://www.whocc.no/ atc_ddd_publications/guidelines/ (accessed January 2014).

20. World Health Organization (2014) WHO's cancer pain ladder for adults. http://www.who.int/cancer/palliative/ painladder/en/ (accessed January 2014).

21. Vargas-Schaffer G (2010) Is the WHO analgesic ladder still valid? Can Fam Pbysician 56, 514-517.

22. McBeth J, Pye SR, O'Neill TW et al. (2010) Musculoskeletal pain is associated with very low levels of vitamin $\mathrm{D}$ in men: results from the European Male Ageing Study. Ann Rheum Dis 69, 1448-1452.

23. Mozaffari-Khosravi H, Nabizade L, Yassini-Ardakani SM et al. (2013) The effect of 2 different single injections of high dose of vitamin D on improving the depression in depressed patients with vitamin D deficiency: a randomized clinical trial. J Clin Psychopharmacol 33, 378-385.

24. Bozkurt NC, Karbek B, Ucan B et al. (2013) The association between severity of vitamin D deficiency and Hashimoto's thyroiditis. Endocr Pract 19, 479-484.

25. Warner AE \& Arnspiger SA (2008) Diffuse musculoskeletal pain is not associated with low vitamin D levels or improved by treatment with vitamin D. J Clin Rheumatol 14, 12-16.

26. Daniel D \& Pirotta MV (2011) Fibromyalgia - should we be testing and treating for vitamin D deficiency? Aust Fam Physician 40, 712-716.

27. Straube S, Derry S, Moore RA et al. (2010) Vitamin D for the treatment of chronic painful conditions in adults. Cochrane Database Syst Rev issue 1, CD007771. 